\title{
On Uncontrolled System Separation in Power System Restoration
}

\author{
F. Edström, L. Söder, Royal Institute of Technology, Stockholm, Sweden
}

\begin{abstract}
Since extended power outages causes severe stress on society and the restoration following a black out is a complex and time consuming task, efficient restoration strategies are needed. During the restoration, the power system undergoes continual changes and therefore it is subject to desired and undesired operations and failures which might be devastating. The main contribution of this paper is a new method to estimate the risk of an uncontrolled system separation in case of a loss of a transmission line or a transformer during a restoration process. The method in this paper assigns failure rates to the transformers and the transmission lines. Further, a graph theoretical method are used to find probability bounds for an uncontrolled system separation for a whole restoration sequence. In a numerical example are three restoration strategies applied to an IEEE 14 bus test system and evaluated. It is shown in the numerical example that the restoration strategy has an high impact on the probability for a system separation.
\end{abstract}

Index Terms-Black out, Restoration, Uncontrolled System Separation, Graph Theory

\section{NOTATION}

The main notation used throughout the paper is stated below for quick reference. Other symbols are defined as required.

$\begin{array}{ll}i & \text { substation or transformer } \\ i^{\prime} & \text { transmission line } \\ j & \text { restoration step } j \\ j^{\prime} & \text { set of vertices in restoration step } j \\ j^{\prime \prime} & \text { set of edges in restoration step } j \\ k & \text { state space } \\ G_{j} & \text { graph in restoration step } j \\ G_{f j} & \text { graph which appears after a failure } \\ \lambda_{i^{\prime}} & \text { failure rate transmission line } \\ \mu_{i} & \text { failure rate transformer } \\ \gamma_{j} & \text { failure rate for graph } G_{j} \\ \Gamma(t) & \text { failure rate for restoration sequence }\end{array}$

\section{INTRODUCTION}

$\mathbf{L}$ ARGE and extended blackouts are rare events which have a huge impact and cause severe stress on the society. Even though much effort is taken to avoid them, they occur from time to time. The restoration process following a large black out is a complex and time consuming task, involving many actors and the need for efficient restoration strategies is obvious. The time it takes to restore a system is critical since important equipment runs on backup power. For example, a remote control of circuit breakers depends for example on backup power at substations. If the power outage at a substation is prolonged, the backup power might be insufficient and the circuit breakers have to be operated manually, which are more time consuming than a remote control. The communication between important actors in the restoration process depends on backup power as well. If the equipment used for communication loses power the restoration will become even more time consuming. A risk which could cause extended power outage is a failure in a component, such as a transformer or a transmission line, during the restoration. If the fault results in an uncontrolled separation of the power system, there might be an unbalanced load and generation situation where frequency control could be difficult. If the fault is not quickly corrected it may result in a new black out where the restoration has to be restarted. If the restoration has to be restarted there will be less backup power available, for example at the substations, and the system has to be restored faster. A fast restoration with less backup power available is obviously associated with higher risks and a lower probability of succeeding.

Power system restoration is an area extensively covered in the literature. See for example, [1] by M.M. Adibi et al. and [2] by D. Lindenmeyer et al. which provides an excellent introduction to the field. Furthermore, a good overview of reliability assessment in power system is given in [3] by R. Billinton et al. Research on controlled power system separation has been conducted in [4] by M.M. Adibi et al. The paper attempts to verify that the location for the uncontrolled separation is independent of the location of the initial fault and dependent of the network topology and load level.

One strategy in case of a black out is to open all circuit breakers $(\mathrm{CB})$ in the transmission network and all CB towards distribution networks. This strategy is known as the "all open" strategy. Its advantage is that the status of the CB is well defined when the restoration starts. An alternative strategy is to open only selected CB's, this strategy is known as "controlled operation" strategy, [5], [6]. Whichever strategy chosen, when rebuilding the power system it is desirable to have reliable restoration sequence. Research on finding suitable restoration paths has been presented by D.S. Kirschen et al. in [7], Park Young-Moo et al. in [8] and by Wang Chong et al. in [9]. However, none of these works treats the fact that a loss of a component during a restoration might result in an uncontrolled system separation. To expand the research in this field new methods considering this risk is needed. To extend the research in this area, a method to analyze the impact of remote control failure on power system restoration time due to battery undersizing was presented by the authors in [10]. However, no consideration is taken to the possibility for a failure in a 
component during the restoration.

This paper presents a new method to evaluate the risk of uncontrolled system separation during a restoration process. The method assigns failure rates to transformers and transmission lines. To characterize the reliability of a power system the topology is represented by a graph with unreliable components. Since the power system changes structure in time during a restoration, all topologies encountered can be used to determine the reliability in terms of system separation for a particular restoration sequence. This paper is structured into five sections. In section III, an overview of the restoration process is given. Section IV explains the approach. Section $\mathrm{V}$ shows how to find the failure rate in terms of system separation. A numerical example is presented in section VI and section VII provides conclusions.

\section{RESTORATION PROCESS}

Power system restoration involves a broad diversity of tasks which depends on the nature of the black out. But the restoration procedure following a power system outage is commonly divided into three major steps [11], see Fig 1. These steps can further be divided into actions and iterations in the restoration process. The first step is to black start the first generators and add critical load or to add cranking power to the non black start generators. The second step refers to the integration of generation units and transmission lines to achieve a target system. During the second step of the restoration of the power system an operator needs to systematically verify different criteria to determine the sequence in which the transmission lines should be energized [12]. These criteria include active and reactive power balance, thermal constrains on transmission lines, sustained overvoltage, switching surges, unstable phenomenon of self-excitation, maintaining steadystate and transient stability during restoration. The energizing of transmission lines is carried out by remote control of circuit breakers which depends on local backup power. Furthermore, the third step aims to minimize the unserved customer loads.

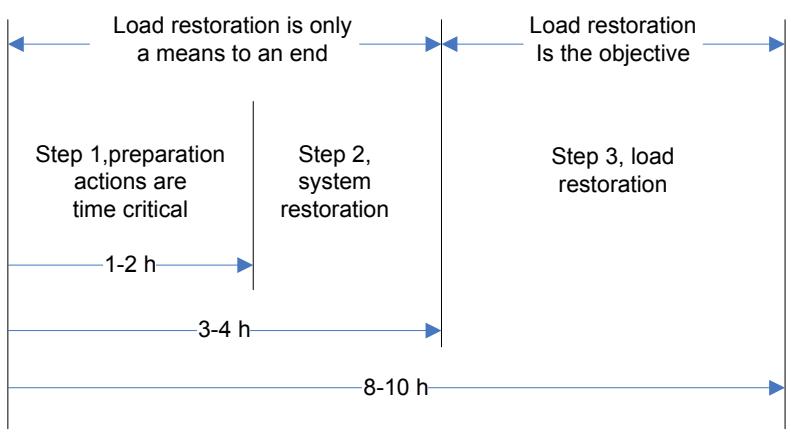

Fig. 1. Overview of the restoration process

A method to estimate the probability for an uncontrolled system separation is specifically applicable in step 2 of the restoration when choosing in which order to energize transmission lines and substations.

\section{APPROACH}

A loss of a generator during the restoration will not instantly cause a system separation, however the loss could cause an overload or a failure somewhere else in the system which in turn could cause an uncontrolled system separation. Due to this fact, the topology of the power system refers to the structure of the substations, transformers and transmission lines. To estimate the probability for an uncontrolled system separation each component is assigned a failure rate. The failure rate for the components could for example be dependent on weather conditions, how fast the restoration proceeds and loading in the system. However, in this paper it is assumed that the components has a constant failure rate. An overview of the method to estimate the probability for an uncontrolled system separation is presented in Fig. 2.

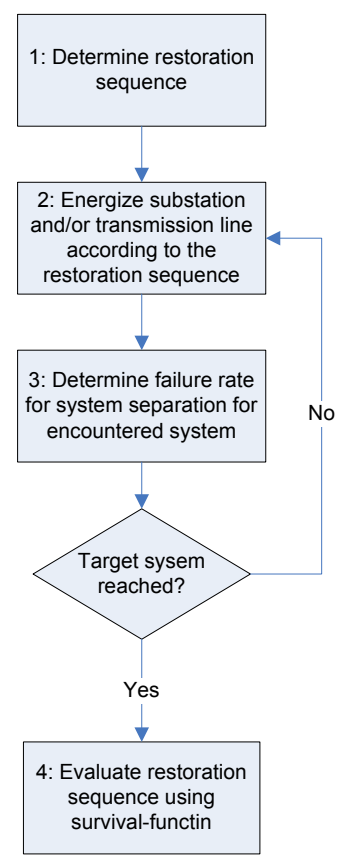

Fig. 2. Overview of method to estimate the reliability for a restoration sequence

The first step is obviously to choose which restoration sequence to evaluate. A restoration sequence in this paper refers to the order in which the transmission lines are used to energize substations and to mesh the system. In the second step an iteration starts where transmission lines are energized according to the picked restoration sequence in an attempt to reach the target system. In the third step, the topology of the encountered power system is represented by a graph. By using graph theory with unreliable components the failure rate in terms of system separation can be estimated. A further explanation of this step is given in section V. In the last step, step 4 , when the failure rate for all encountered system is known the restoration sequence can be an evaluated in terms of probability for an uncontrolled system separation during the restoration. 


\section{DETERMINE FAILURE RATE FOR SYSTEM SEPARATION}

A proposal for step three and four in the method presented is given in this section. The topology of a power system can be represented by a graph. The graph can in turn be used to characterize the systems reliability. The standard terminology of graph theory, as it is introduced in most text books, is used. Let $G=(V, E)$ be an directed graph where the edges has arbitary directions and where substations and transformers are represented by vertices and transmission lines as edges. In case of a black out, some or in worst case all substations and transmission lines are no longer energized. Let the set $V_{1}$ and $E_{1}$ denote the set of substations and transmission lines which are not energized after the black out. The topology of the power system after the black out is then given by $G_{1}=\left(E \backslash E_{1}, V \backslash V_{1}\right) . G_{1}$ is therefore the starting point for the restoration. See Fig. 3 and Fig. 4 for an example.

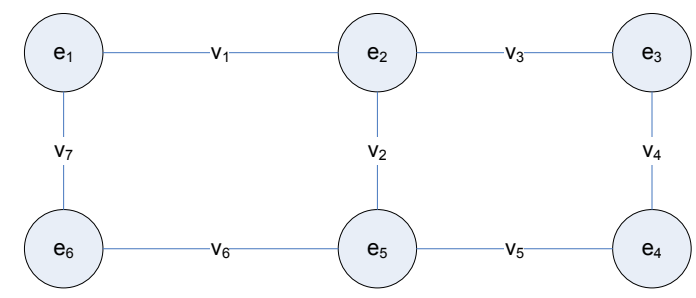

Fig. 3. Example of a topology of a power system given as a graph, $G=$ $(E, V)$.

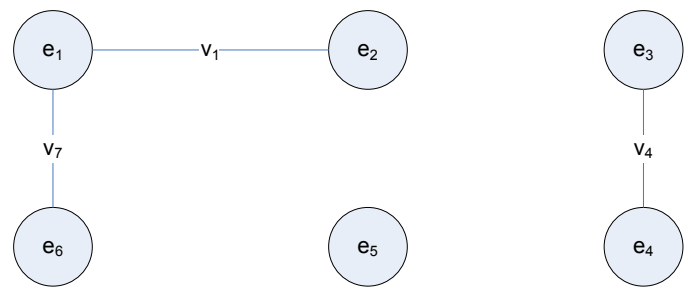

Fig. 4. Example of a topology of a power system after a disturbance where $E_{1}=\left\{e_{5}\right\}, V_{1}=\left\{v_{3}, v_{5}, v_{6}\right\}$ and $G_{1}=\left(E \backslash E_{1}, V \backslash V_{1}\right)$

If either $V_{1}$ or $E_{1}$ is a cut set the power system has split into two or more isolated systems. In the restoration process, the analogy to energize lines and substations by operating circuit breakers is to introduce edges and vertices in a certain order from $V_{1}$ and $E_{1}$ into $G_{1}$ to rebuild the original system $G$. When energizing substations $i$ using transmission line $i^{\prime}$ the notations used are

$$
\begin{gathered}
V_{j^{\prime}+1}=V_{j^{\prime}} \backslash v_{i} \\
E_{j^{\prime \prime}+1}=E_{j^{\prime \prime}} \backslash e_{i^{\prime}}
\end{gathered}
$$

Using this notation $E_{n}=E, V_{m}=V$ and $G_{j}=$ $\left(E \backslash E_{j^{\prime \prime}}, V \backslash V_{j^{\prime}}\right)$ where $n$ is the number of transmission lines in the system (or edges in the graph) and $m$ is the number of substations (or vertices in the graph). To clarify, the system topologies which are encountered during the restoration are

$$
G_{1} \subset \ldots \subset G_{j} \subset G_{j+1} \ldots \subset G
$$

which all has there own failure rate in terms of system separation which depends on their system configuration. As stated in the indroduction, the intation of the paper is to propose a method which estimates the risk for a system separaton for a whole restoration sequence.

\section{A. System separation}

If one or several transmission lines and/or transformers fails in a system whit a topology represented by $G_{j}$, a new system with a new topology appears. Let this system be denoded by $G_{f j}$. The question is if the failure, or the failures, has caused a system separation.

To formalize, let $\lambda$ and $\mu$ denote failure rates for the transmission lines and the transformers respectivitly. For simplicity, let $\lambda_{i^{\prime}}=\lambda\left(e_{i^{\prime}}\right)$ for $1, \ldots, n$ and $\mu_{i}=\mu\left(v_{i}\right)$ for $1, \ldots, m$. Let $V_{f j}$ and $E_{f j}$ denote the set of transmission lines and transformers that fails at restoration step $j$. Note that if the failure rate is small, it is likely that there will only be one failure which means that it is likely that $V_{f j}$ or $E_{f j}$ will only contain one edge or node. However this does not exclude the risk for more failures. The topology for system $G_{j}$ after it is subjected to a failure is given by

$$
G_{f j}=\left(E \backslash\left\{E_{j^{\prime \prime}} E_{f j}\right\}, V \backslash\left\{V_{j^{\prime}} V_{f j}\right\}\right) .
$$

Moreover, let $c(G)$ be the number of components of a graph $G$. If a transmission lines trips, or there is a failure in a transformer, the system will be subjected to a separation if

$$
c\left(G_{j}\right)<c\left(G_{f j}\right)
$$

e.g. an uncontrolled system separation has occurred due to the failure. Let the failure rate, in terms of system separation, for $G_{j}$ be given by $\gamma_{j}=\gamma\left(G_{j}\right)$. Obviously, it depends on the topology and the failure rates for the transmission lines, $\lambda$, and the transformers, $\mu$. Since the system undergoes changes, from $G_{1}$ to $G_{2}$ and so on until $G$, which each has a failure rate $\gamma_{1}, \ldots, \gamma_{n}$. Let

$$
\Gamma(t)=\left\{\begin{array}{cc}
\gamma_{1} & 0<t<h_{1} \\
\cdot & \\
\cdot & \\
\cdot & \\
\cdot & \\
\gamma_{n} & h_{n-1}<t<h_{n} .
\end{array}\right.
$$

It is well known in reliability theory that the survival function, which gives the probabilty that the system has not faced a system separation in this case, is given by

$$
R(t)=1-F(t)=e^{-\int_{0}^{t} \Gamma(u) d u}
$$

where $F(t)$ is the cumulative distribution function on $[0, \infty)$. Therefore, if $\Gamma(t)$ is determined, restorations sequences can be evaluated in terms of probability for a system separation due to a failure when rebuilding the system. 


\section{B. Determine failure rate $\gamma_{j}$ for the system $G_{j}$}

The probability that a component, for example the transformer, will fail during time interval, $\left(h_{j}, h_{j+1}\right]$ given that it works at time $h_{j}$, is given by

$$
P\left(\Delta t+h_{j}>T>h_{j} \mid T>h_{j}\right) \approx \mu_{j} \Delta t_{j}
$$

for small $\Delta t_{j}$ where

$$
\Delta t_{j}=h_{j+1}-h_{j} .
$$

Since Eq. 8 gives the probability that component will fail, algorithms, for example [13], designed to evaluate the reliability of networks, can be used to determine failure rate in terms of system separation. Hence, let

$$
\begin{aligned}
p_{i^{\prime}} & =\lambda \Delta t \\
p_{i} & =\mu \Delta t
\end{aligned}
$$

Due to the complexity when studying the performance of large graphs with failure prone components, two compromises are usually made. The first compromise is to deal with reliability bounds instead of exact solutions. The second compromise is to simplifying the analysis with perfect reliable components. Li and Silvester [13] has presented an algorithm named ORDER which evaluates the $l$ most probable state spaces and forms reliability bounds using this state spaces. The algorithm has computational complexity $O\left(n_{0}^{2} l+n_{0} l \log l\right)$ where $n_{0}$ is the amount of failure prone components in the system. Since it is proven to converge fast and relativity simple to work with, it is suitable for determine the reliability bounds for the failure rate $\gamma_{j}$. A state of a system is identified by its failed components. The possible states of a system, $G_{j}$, is denoted $S_{k j}$ where $k=1, \ldots, 2^{l}$. It is not unreasonable to assume a constant failure rate for the transmission lines or for the transformers since the restoration process is relativity short in time. Hence, the probability for state $S_{k j}$ is then given by

$$
\begin{aligned}
P\left(S_{k j}\right)= & \prod_{i \in E_{f j}} p_{i} \prod_{i \notin E_{f j}}\left(1-p_{i}\right) \prod_{i^{\prime} \in V_{f j}} p_{i^{\prime}} \\
& \prod_{i^{\prime} \notin V_{f j}}\left(1-p_{i^{\prime}}\right) .
\end{aligned}
$$

It is not unreasonable to assume that $p_{i}<<1 / 2$ and $p_{k}<<$ $1 / 2$ which makes $S_{1 j}$ the most probable state spaces with only working components. The second most probable state space is the one where the most unreliable component has failed. However, the next state space is more complex to predict. The $l$ most probable state spaces at restoration step $j$ are then given by the algorithm ORDER or any other suitable algorithm.

In order to find the bounds for the failure rate, each state space has to be evaluated if Eq. 5 is valid or not. In spectral graph theory, the number of components in a graph is equal the number of times zero appears as an eigenvalue of the Laplacian matrix to the system. If $A\left(G_{j}\right)$ is the incident matrix, it is well known that the Laplacian matrix is given by

$$
Q\left(G_{j}\right)=A\left(G_{j}\right) A\left(G_{j}\right)^{T} .
$$

Let $\eta\left(G_{j}\right)$ be the multiplicity of zero as an eigenvalue to the associated Laplacian matrix $Q\left(G_{j}\right)$. Furthermore, each state has to be evaluated if the failure has caused an uncontrolled system separation or not according to Eq. 5. Let

$$
C_{k j}\left(S_{k j}\right)=\left\{\begin{array}{cc}
0 & \text { if } \eta\left(G_{f j}\right)>\eta\left(G_{j}\right) \\
1 & \text { if not. }
\end{array}\right.
$$

When averaging $C_{k j}$ over all possible state spaces, $k, E\left(C_{j}\right)$ is determined. $E\left(C_{j}\right)$ is the probability that the system will not face an uncontrolled system separation during the restoration. However, since large system is considered reliability bounds are used. The bounds for $E\left(C_{j}\right)$ is given by

$$
\begin{aligned}
C_{L j}= & \sum_{k=1}^{m} P\left(S_{k j}\right) C_{k j}\left(S_{k j}\right) \\
C_{U j}= & \sum_{k=1}^{m} P\left(S_{k j}\right) C_{k j}\left(S_{k j}\right)+ \\
& \left(1-\sum_{k=1}^{m} P\left(S_{k j}\right)\right) .
\end{aligned}
$$

As stated earlier, it is not unreasonable to assume a constant failure rate since the time spent in each restoration step is relative small. Since the failure rate is assumed to be constant the boundaries for the failure rate is found from

$$
\begin{gathered}
\gamma_{L j}=\frac{C_{L j}}{\Delta t_{j}} \\
\gamma_{U j}=\frac{C_{U j}}{\Delta t_{j}}
\end{gathered}
$$

To evaluate a restoration sequence for $j=1, \ldots, m$ Eq. 7 is used.

\section{Computational complexity}

Since each component can be either in failure mode or working mode, the number of state spaces are $2^{n_{0 j}}$ where $n_{0 j}$ is the number of failure prone components in graph $G_{j}$. If it is assumed that all transmission lines and all substations are assigned a failure rate, the case where the number of state spaces increases the least is when a transmission line is used to mesh the system since only one new failure prone component is introduced into the graph. Hence, the minimum number of state spaces, $S_{N}$, in the restoration sequence is given by

$$
S_{N} \geq \sum_{j=1}^{n} 2^{j}=2^{n+1}-1
$$

In the worst case, the number of failure prone components increases with two for every transmission line. This is the case when a transmission line is used to energize a substation. Hence, the number of state spaces will always be less than

$$
S_{N} \leq \sum_{j=1}^{n} 2^{2(j+1)}
$$




\section{NUMERICAL EXAMPLE}

To evaluate the reliability for some restoration sequences a IEEE 14 bus test system which represents a part of the American Electric Power System in the Midwestern US as of February 1962 is used, see Fig 5 for an overview. It is assumed that the restoration process has reached step 2 in accordance to Fig. 1 and [11]. It is further assumed that all CB are open in accordance to the all open strategy in [5] and [6]. An operator has hence to decide in which order to energize the transmission lines and the substations. Since all equipment runs on backup power during a blackout it is desirable to energize all substations as quick as possible to reduce the risks associated with limited backup power, such as remote control failure. The intention of this numerical example is to show the impact on the probability for an uncontrolled system separation of such restoration process.

The topology of the system given in graph format is presented below in Fig 6. In Fig 6, each transmission line in the system has been given a number. This number is used to present the restoration sequences in table II. It can be discussed if transmission lines $8,9,10,11,12,13$ and 14 really are transmission lines or just connections within a substation. In this example, they are considered as a component such as a transmission line and assigned a failure rate. The failure rates used in the study are given in table I. Normally failure rates for transmission lines are given in failure rate per length. However, to simplify the numerical example it is assumed that all transmission lines have the same failure rate.

TABLE I

FAILURE RATES

\begin{tabular}{ll}
\hline Component & Failure rate \\
\hline Transmission lines & 0.01 \\
Transformers & 0.05 \\
\hline
\end{tabular}

\section{A. Restoration Sequences in the System Restoration Step}

1) One strategy is to energize all substations using a minimum number of $\mathrm{CB}$ operations. When all substations are energized additional transmission lines are used to increase the transmission capacity and to increase the reliability of system. This strategy will have a high failure rate in the beginning but it will energize all substations quickly. This strategy is named strategy one.

2) An alternative restoration sequence is to build a strong well connected system from the beginning. When using this strategy it will take more time to energize all substations and it is therefore a higher probability that there will be problem associated with backup power. This restoration sequence will obviously have a lower failure rate but it will take more time to energize all substations. This strategy is named strategy two.

3) A third restoration sequence is a combination of the two mentioned. It is taken into account that the substations have to be energized quickly when building a well connected system from the beginning. This strategy is named strategy three.

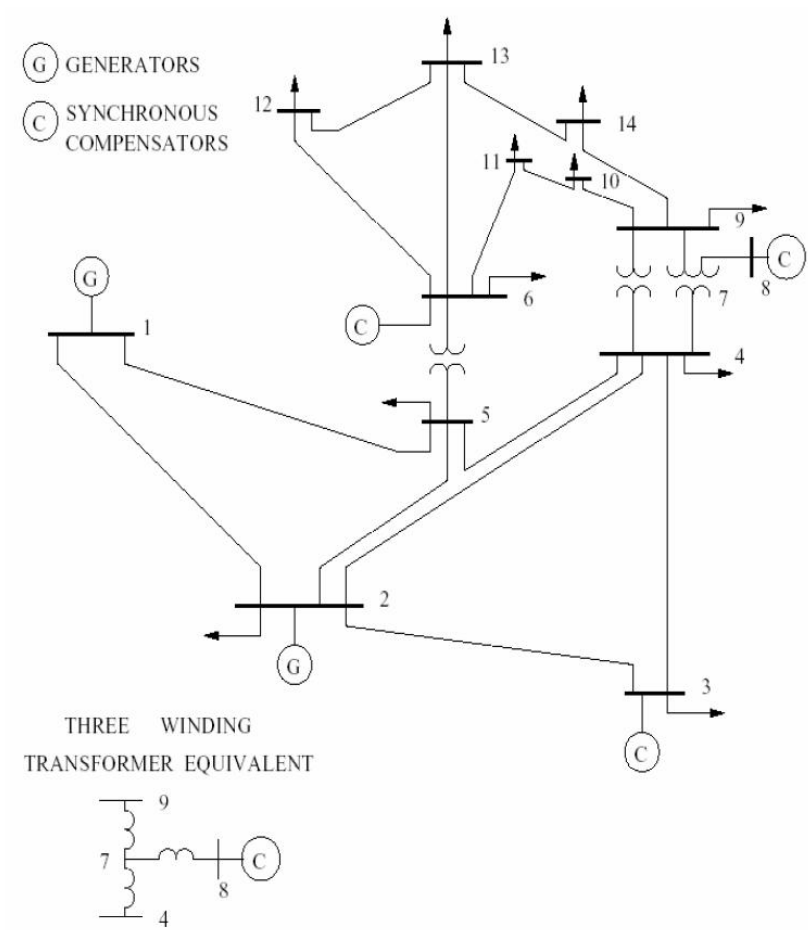

Fig. 5. IEEE 14 bus test system which represents a part of the American Electric Power System in the Midwestern US as of February 1962

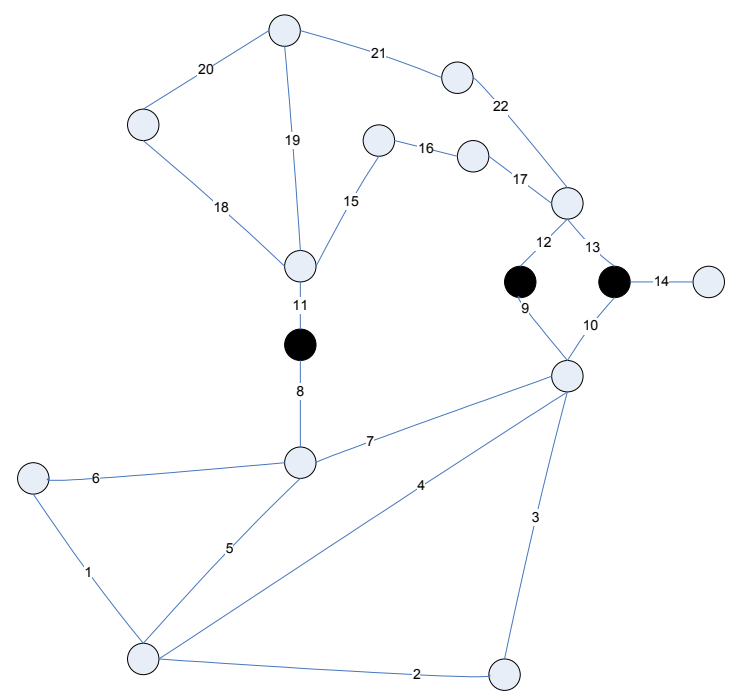

Fig. 6. The topology of the American Electric Power System in the Midwestern US as of February 1962

The following three restoration sequences presented below in table II are used in the numerical example. In this numerical example it is assumed that the time spent in every stage is equal and assumed to be 1 time unit. Further, it is assumed that the generator connected to bus 1 is a black start generator. The restoration starts therefore from bus 1 . The system integration of generators and load is neglected since the interest is to decide the probability for a system separation.

To demonstrate the three different restoration strategies fig $7,8,9$ shows restoration one, two and three respectively when 10 transmission lines has been energized. 
TABLE II

THE RESTORATION SEQUENCES USED IN THE NUMERICAL EXAMPLE

\begin{tabular}{ll}
\hline Strategy & Sequence \\
\hline One & 123912222120181516 \\
& $118101445671317 \quad 19$ \\
Two & 15674239121310811 \\
& 151617182019212214 \\
Three & $12376811 \quad 1820212212$ \\
& 91716151014135419 \\
\hline
\end{tabular}

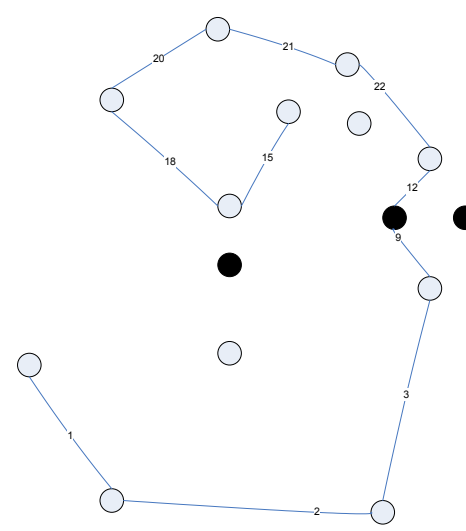

Fig. 7. Restoration sequence one when 10 transmission lines has been energized

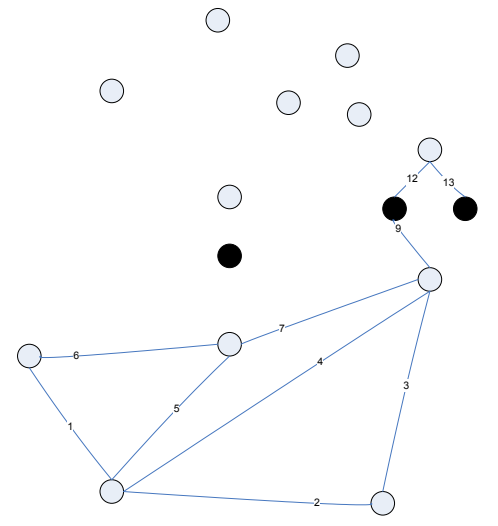

Fig. 8. Restoration sequence two when 10 transmission lines has been energized

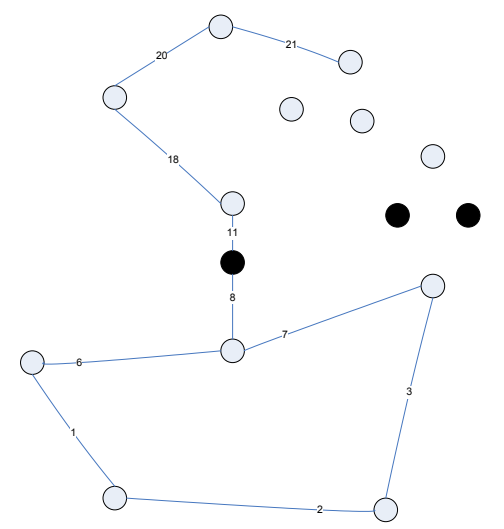

Fig. 9. Restoration sequence three when 10 transmission lines has been energized
Note that the numbers of not energized substations are 4, 8 and 6 respectively. It is clear that restoration sequence one has manage to energize the most number of substations using the same number of transmission lines. However, a loss of any transmission line will instantly cause a system separation at this stage. As a contradiction, a loss of transmission line 1 to 7 in restoration sequence two or 1,2, 3, 6 and 7 in restoration sequence three will not cause an instant system separation. In figure 13 it is seen that the choice of restoration sequence significantly affect the probability of facing an uncontrolled system separation. Moreover, the most critical configuration encountered for a restoration sequence can be identified from the figures showing the failure rates. For example, in restoration sequence one the most critical configuration is reached after energizing 9 transmission lines. However, the failure rate drops significantly when transmission line number 15 is energized and the system forms a closed loop.

\section{B. Results}

Figure 10, 11 and 12 shows the failure rate $\Gamma(t)$ for restoration sequence one, two and three respectively. The survival function for the restorations sequences is presented in figure 13.

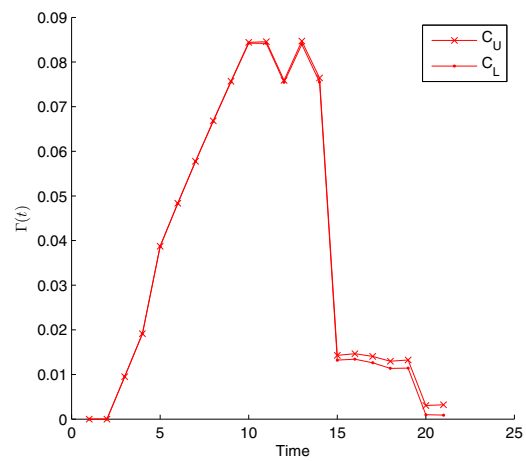

Fig. 10. The failure rate for system separation, $\Gamma(t)$, for restoration sequence one

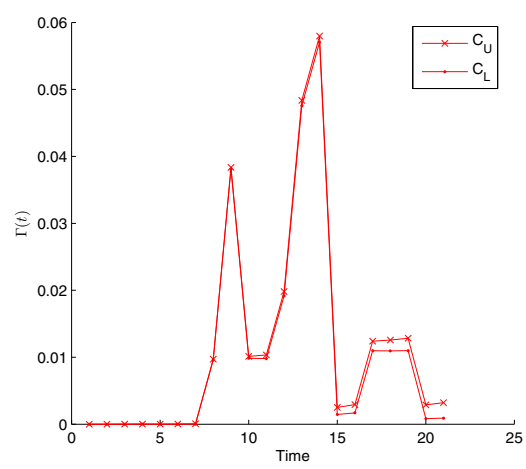

Fig. 11. The failure rate for system separation, $\Gamma(t)$, for restoration sequence two 


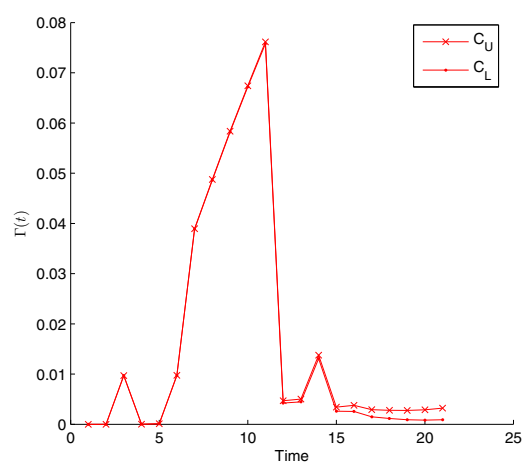

Fig. 12. The failure rate for system separation, $\Gamma(t)$, for restoration sequence three

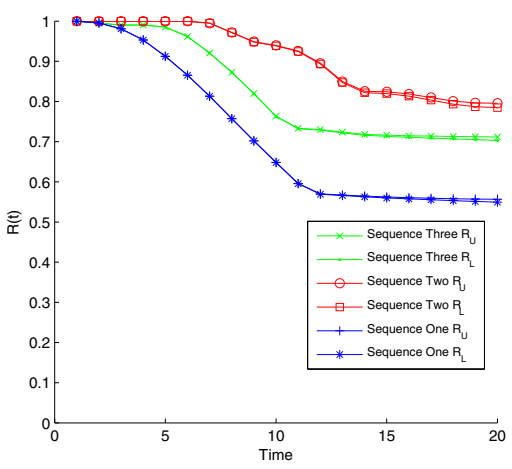

Fig. 13. The survival function, $R(t)$, for the three different restoration sequences. Restoration sequence one is marked with dots, restoration sequence two is given marked plus and restoration sequence three is marked with crosses.

\section{CONCLUSION}

A method to estimate the risk for an uncontrolled system separation in case of a failure on a transmission line has been presented. The method can be used in restoration planning to evaluate different restoration strategies. Furthermore, the method has been applied in a numerical example where three different restoration strategies have been evaluated. In the numerical example, it is showed that the restoration sequence has an impact on the reliability of not loosing connectivity during the restoration. Furthermore, in the numerical example it is more time consuming to energize all substation if a reliable restoration sequence is chosen. This might increase the risks associated with backup power. If the power outage is extended a less reliable restoration sequence may has to be used. If this is the case it is necessary to understand the risks when dealing with fast restorations.

The method can be used in restoration planning to determine which sequence that is most likely to succeed is given as a conclusion. It is also showed that the number of operations needed to find $\Gamma$ for a particular restoration sequence when using the algorithm is bounded by $2^{n+1}-1 \leq S_{N} \leq \sum_{j=1}^{n} 2^{2(j+1)}$

\section{REFERENCES}

[1] M. M. Adibi, editor. Power System Restoration. IEEE Press, 2000.
[2] D. Lindenmeyer, H.D. Dommel and M.M. Adibi. "Power System Restoration - a bibliographic survey". Electrical Power and Energy Systems, Volume: 23, Issue: 3, Pages: 219 - 227, 2001.

[3] R. Billinton, R.N. Allan and L. Salvaderi, editor. Applied Reliability Assessment in Electric Power Systems. IEEE Press, 1991.

[4] M.M. Adibi,R.J. Kafka, S. Maram, L.M. Mili. "On Power System Controlled Separation". IEEE Transactions on Power Systems, Volume: 21, Issue: 4, Pages: 1894 - 1902, 2006.

[5] M. M. Adibi and R. J. Kafka. "Power System Restoration Issues". IEEE Computer Applications in Power, Volume: 4, Issue: 2, Pages: 19 - 24 , 1992.

[6] M.M. Adibi, L.H. Fink, C.J. Andrews, F. Arsanjani, M.W. Lanier, J.M. Miller, T.A. Volkmann and J. Wrubel. "Special Considerations in Power System Restoration". IEEE Transanaction on Power Systems, Volume: 12, Issue: $11,1992$.

[7] D.S. Kirschen and T.L. Volkmann. "Guiding a Power System Restoration with an Expert System". IEEE Transactions on Power Systems, Volume: 6, Issue: 2, Pages: 558 - 566, 1991.

[8] Park Young-Moon and Lee Kwang-Ho. "Application of Expert System to Power System Restoration in Sub-Control Center ". IEEE Transactions on Power Systems, Volume: 12, Issue: 2, Pages: 629 - 635, 1997.

[9] Wang Chong, V. Vittal, V.S. Kolluri and S. Mandal. "PTDF-Based Automatic Restoration Path Selection ". IEEE Transactions on Power Systems, Volume: 25, Issue: 3, Pages: 1 - 1, 2010.

[10] F. Edström and L. Söder. "Impact of Remote Control Failure on Power System Restoration Time". IEEE Conference on Probabilistic Methods Applied to Power System, Pages: 343 - 347, 2010.

[11] M.M. Adibi and L.H. Fink. "Power System Restoration Planning". IEEE Transaction on Power Systems, Volume: 9 , Issue: 1, Pages: 22 - 28, 1994.

[12] M.M. Adibi, W. Alexander, and B.Avramovic. "Overvoltage Control During Restoration ”. IEEE Power Engineering Review, Volume: 12, Issue: 11, 1992.

[13] Y. Lam and V. Li. "An Improved Algorithm for Performance Analysis of Networks with Unreliable Components". IEEE Transactions on Communications, Volume: 34 , Issue: 5, Pages: 496 - 497 , 1986. 